\title{
Matched Comparison of Two Different Biological Prostheses for Complete Supra-annular Aortic Valve Replacement
}

\author{
Kathrin Fiegl ${ }^{1} \quad$ Marcus-Andre Deutsch ${ }^{1}$ Ina-Christine Rondak ${ }^{2}$ Ruediger Lange ${ }^{1,3}$ Ralf Guenzinger ${ }^{1}$ \\ ${ }^{1}$ Department of Cardiovascular Surgery, German Heart Center \\ Address for correspondence Kathrin Fiegl, MD, Department of \\ Munich, Munich, Germany \\ Cardiovascular Surgery, German Heart Center Munich, Lazarettstraße \\ 2 Institute of Medical Statistics and Epidemiology, Technical University \\ 36, Munich 80636, Germany (e-mail: fiegl@dhm.mhn.de). \\ Munich, Munich, Germany \\ 3 Partner Site Munich Heart Alliance, DZHK (German Center for \\ Cardiovascular Research), Munich, Germany
}

Thorac Cardiovasc Surg 2015;63:459-466.

\begin{abstract}
Keywords

- aortic valve and root

- heart valve surgery

- aorta/aortic

Objective The aim of this retrospective study was to evaluate the hemodynamic performance of the St. Jude Medical Trifecta (SJM Trifecta; St. Jude Medical, St Paul, Minnesota, United States) and the Carpentier-Edwards Perimount Magna Ease (CEPM Ease; Edwards Lifesciences, Irvine, California, United States) bioprostheses early postoperative and at 1 year.

Methods From October 2007 to October 2008, a total of 61 consecutive patients underwent aortic valve replacement (AVR) with the CEPM Ease prosthesis. From a prospective cohort of 201 patients (March 2011 to January 2012) who received AVR with the SJM Trifecta valve, a matched group of 51 patients was selected. Matching was conducted $1: 1$ by ejection fraction, gender, age, and body surface area. A Hegar dilator was used to define the aortic tissue annulus diameter. Data were grouped on the basis of the patient's tissue annulus diameter ( $\leq 22 \mathrm{~mm} ; 23-24 \mathrm{~mm} ; \geq 25 \mathrm{~mm}$ ).

Results Early postoperative and at 1 year mean pressure gradients (MPGs) in the various groups ranged from $7.2 \pm 4.6$ to $7.1 \pm 2.4 \mathrm{~mm} \mathrm{Hg}$ and from $10.0 \pm 4.3$ to $8.0 \pm 2.8 \mathrm{~mm} \mathrm{Hg}$ in the SJM Trifecta group and from $18.0 \pm 5.0$ to $12.1 \pm 3.6 \mathrm{~mm} \mathrm{Hg}$ and from $17.7 \pm 4.5$ to $11.8 \pm 3.2 \mathrm{~mm} \mathrm{Hg}$ in the CEPM Ease group, respectively. Likewise, effective orifice areas (EOAs) ranged from $1.7 \pm 0.5$ to $2.0 \pm 0.5 \mathrm{~cm}^{2}$ and from $1.5 \pm 0.3$ to $1.7 \pm 0.4 \mathrm{~cm}^{2}$ in the SJM Trifecta group and from $1.3 \pm 0.5$ to $1.9 \pm 0.5 \mathrm{~cm}^{2}$ and from $1.2 \pm 0.3$ to $1.8 \pm 0.3 \mathrm{~cm}^{2}$ in the CEPM Ease group, respectively. A marked left ventricular mass (LVM) regression across all annulus sizes was noted in both groups. Severe patient-prosthesis mismatch (PPM) was infrequent overall.

Conclusion The SJM Trifecta valve showed lower MPGs early postoperative and at 1 year as well as higher EOA and effective orifice area index early postoperative. No significant differences were detected with regard to LVM regression and PPM.
\end{abstract}

received

September 16, 2014

accepted after revision

January 26, 2015

published online

April 20, 2015 (c) 2015 Georg Thieme Verlag KG

Stuttgart · New York
DOI http://dx.doi.org/ 10.1055/s-0035-1548744. ISSN 0171-6425. 


\section{Introduction}

Surgical aortic valve replacement (AVR) represents the gold standard for patients with calcified aortic stenosis due to its low morbidity and mortality. ${ }^{1}$ In 2013 , almost 12,000 patients underwent isolated aortic valve surgery with the use of extracorporeal circulation in Germany (GSTVS Annual Registry, survey period 2013). Bioprostheses were implanted in 93\% of these patients (GSTVS Annual Registry, survey period 2013), since in older patients the risk-benefit profile is more favorable for bioprosthesis than for mechanical prostheses. ${ }^{2,3}$ The hemodynamic performance of today's third-generation stented pericardial prostheses is comparable to stentless biological valves. ${ }^{4}$ Several studies could demonstrate that supra-annular implantation of stented biological valves provides a larger effective orifice area (EOA), as the stent and the sewing ring are positioned on top of the native annulus. ${ }^{5-8}$ Consequently, their larger EOA results in less patient-prosthesis mismatch (PPM), especially in patients with a small aortic annulus. ${ }^{7,9}$

The SJM Trifecta (St. Jude Medical, St Paul, Minnesota, United States) combines some new construction methods, which should improve hemodynamics. The aim of the study was to validate the SJM Trifecta concept of valve design in comparison with the well-established concept of the Carpentier-Edwards Perimount Magna Ease (CEPM Ease; Edwards Lifesciences, Irvine, California, United States). For this purpose, short-term functional and clinical data after AVR with either the new SJM Trifecta bioprosthesis or the CEPM Ease bioprosthesis were evaluated. Analysis was conducted in a matched patient cohort, and a noncommercial sizer (Hegar dilator) was used to define the aortic tissue annulus diameter to allow for a better comparison of valve performance.

\section{Materials and Methods}

\section{Patients}

Between August 2007 and August 2008, a total of 61 consecutive patients underwent AVR with the CEPM Ease bioprosthesis. Between March 2011 and January 2012, a total of 201 consecutive patients underwent AVR with the SJM Trifecta bioprosthesis. For a better comparison in this retrospective study, the two cohorts were matched, resulting in 51 patients for each group. Matching was conducted $1: 1$ by ejection fraction ( $\mathrm{EF}$ ) ( $>50$ vs. $30-49 \%$ ), gender, age ( \pm 5 years), and body surface area (BSA) $\left( \pm 0.1 \mathrm{~m}^{2}\right)$. Early follow-up was performed within 10 days postoperatively, including transthoracic echocardiography. Transthoracic echocardiography was repeated 1 year after the operation and all valve-related complications were documented at the time of appearance according to the guidelines for reporting morbidity and mortality after cardiac valvular operations. ${ }^{10}$ The study was approved by the local ethics committee (approval reference number: 314/13). All aortic valve lesions as well as combined procedures were admitted.

\section{Transthoracic Echocardiography}

Transthoracic Doppler echocardiography was performed according to the data requirements of the Food and Drug
Administration Replacement Heart Valve Guidance, Version 4.1. ${ }^{11}$

Echocardiographic measurements included the transvalvular and the left ventricular outflow tract (LVOT) mean and maximal flow velocity and gradients. The LVOT diameter was assessed from a parasternal long-axis view using an expanded view. The left ventricular end-systolic diameter (LVESD), the left ventricular end-diastolic diameter (LVEDD), the thickness of the left ventricular posterior wall (LVPWD), and the intraventricular septum (IVS) were assessed in the parasternal view by multiple M-mode measurements.

\section{Hemodynamic Calculations}

The transvalvular mean pressure gradient (MPG) was calculated as the difference of the mean aortic and the mean LVOT gradient.

EOA was calculated according to the continuity equation:

EOA $\left(\mathrm{cm}^{2}\right)=(\text { LVOT diameter } \times 0.5)^{2} \times \pi \times[$ VTI $($ LVOT $) /$ $\operatorname{VTI}($ valve $)]$

Effective orifice area index (EOAI) was calculated by dividing EOA by BSA:

EOAI $\left(\mathrm{cm}^{2} / \mathrm{m}^{2}\right)=\mathrm{EOA} / \mathrm{BSA}$

BSA was calculated according the Du Bois equation ${ }^{12}$ :

BSA $\left(\mathrm{m}^{2}\right)=0.007184 \times$ height $^{0.725}(\mathrm{~cm}) \times$ weight $^{0.425}$ $(\mathrm{kg})$

Left ventricular mass (LVM) was calculated according to Devereux ${ }^{13}$ :

$\mathrm{LVM}(\mathrm{g})=1.04 \times\left[(\mathrm{LVEDD}+\mathrm{LVPWD}+\mathrm{IVS})^{3}-\right.$

$\left.(\mathrm{LVEDD})^{3}\right]-13.6$

LV hypertrophy was defined as present with an LVM index greater than $131 \mathrm{~g} / \mathrm{cm}^{2}$ in men and greater than $100 \mathrm{~g} / \mathrm{cm}^{2}$ in women. ${ }^{14}$

PPM was defined as not present for EOAI greater than $0.85 \mathrm{~cm}^{2} / \mathrm{m}^{2}$, moderate for EOAI between 0.65 and 0.85 $\mathrm{cm}^{2} / \mathrm{m}^{2}$, and severe for EOAI less than $0.65 \mathrm{~cm}^{2} / \mathrm{m}^{2} .15$

\section{Implanted Bioprosthesis}

Carpentier-Edwards Perimount Magna Ease Prosthesis The CEPM Ease is the most recent stented biological prosthesis for complete supra-annular implantation developed by Edwards Lifesciences. The valve implements a lower profile stent than its precursor, the Carpentier-Edwards Perimount Magna prosthesis (i.e., $1 \mathrm{~mm}$ lower for valve size 21). The leaflets are made of bovine pericardial tissue, chemically stabilized with glutaraldehyde, and are attached to a flexible cobalt-chromium stent. The Edwards ThermaFix technology is used to protect from calcification. CE certification was achieved in 2006. The CEPM Ease bioprosthesis has already been shown to generate excellent hemodynamic and clinical data. ${ }^{9,16}$

\section{St. Jude Medical Trifecta Prosthesis}

The SJM Trifecta is a trileaflet stented pericardial valve for supra-annular implantation in the aortic position. The SJM Trifecta stent consists of titanium with vertical stent posts. The stents are covered in polyester fabric and porcine pericardium and the leaflet stitching is controlled at the top of the 
stent posts. The leaflets, made of bovine pericardium, are mounted exteriorly to the stent posts. Both pericardial tissues (bovine and porcine) are chemically stabilized in glutaraldehyde. The LinxAC technology is used to protect from calcification. CE certification was achieved in March 2010.

\section{Implantation Technique}

AVR was performed through a complete or partial upper sternotomy using standard cardiopulmonary bypass under mild hypothermia $\left(32^{\circ} \mathrm{C}\right)$ with cold crystalloid cardioplegia and topical surface cooling. After removal of the native aortic valve and decalcification of the annulus, the aortic annulus diameter was first determined by inserting a Hegar dilator and then the valve size was determined using the valve sizer provided by the manufacturer. Both prostheses were implanted with pledged, interrupted, noneverting mattress sutures.

All patients were discharged on a regimen of phenprocoumon (Coumadin) for the first 3 months postoperatively.

\section{Statistical Analysis}

Categorical variables are presented as frequencies and continuous variables as mean \pm standard deviation, or medians and interquartile ranges for variables with skewed distributions. Accounting for the matched design, group comparisons are computed by the use of the paired $t$-test, the Wilcoxon signed-rank test, the McNemar test or Bowker test, as appropriate. For explorative subgroup analysis according to annulus diameter, tests for independent samples (i.e., $t$-test and Mann-Whitney $U$ test) are used. Associations between independent categorical variables are evaluated using Fisher's exact test. The Kaplan-Meier method was used to estimate rates of overall survival rate, which are presented with $95 \%$ confidence intervals (CIs). All reported $p$-values are twotailed, with a significance level of 0.05 , and have not been adjusted for multiple testing. Statistical analyses were performed with the Statistical Package for the Social Sciences version 20.0 (SPSS Inc., Chicago, Illinois, United States).

\section{Results}

\section{Patients}

Follow-up was 99\% complete. One patient of the CEPM Ease group quitted the study for personal reasons. As the patients were matched, distributions of age, gender, BSA, and EF were similar in both groups ( - Table 1). Baseline characteristics and New York Heart Association (NYHA) classification are depicted in - Table 2.

\section{Operative Data}

Overall 30-day mortality was $0 \%$ in the SJM Trifecta group and $2.0 \%$ ( 1 patient) in the CEPM Ease group. This one patient died of multiple organ failure following septicemia, and the death was not valve related in as much as endocarditis was excluded by necropsy. Kaplan-Meier survival curves at 1 year after the operation are depicted in -Fig. 1. In one case, the CEPM Ease valve was explanted intraoperatively because of a ventricular septal defect (VSD), which was accidentally created by removing a big chunk of calcium. A suitable, one size smaller Carpentier-Edwards Perimount valve was implanted after VSD closure. Operative data are depicted in -Table 3.

\section{Survival}

Overall survival at 1 year in the CEPM Ease group and in the SJM Trifecta group were 90.6\% (95\% CI [81.1\%; 100\%]) and $96.0 \%$ (95\% CI [90.8\%; 100\%]), respectively. There were three deaths during follow-up in the CEPM Ease group. Two deaths were valve related due to prosthetic valve endocarditis. One patient died of serious peripheral artery occlusive disease. In the SJM Trifecta group, there were no valve-related deaths during follow-up. However, one patient died from an accident and another one due to a metastatic carcinoma of the prostatic gland.

\section{Adverse Events}

The McNemar test showed no statistically significant differences in adverse events between the two groups ( $p=0.359$ ).

Table 1 Matching

\begin{tabular}{|l|l|l|}
\hline & CEPM Ease $(\boldsymbol{n}=\mathbf{5 1})$ & SJM Trifecta $(\boldsymbol{n}=\mathbf{5 1})$ \\
\hline Age $^{\mathrm{a}}(\mathrm{y})$ & $66.0 \pm 7.1$ & $66.3 \pm 6.9$ \\
\hline Gender & & 40 \\
\hline Male $(n)$ & 40 & 11 \\
\hline Female $(n)$ & 11 & $171.5 \pm 7.4$ \\
\hline Height $^{\mathrm{a}}(\mathrm{cm})$ & $173.6 \pm 7.9$ & $82.5 \pm 13.3$ \\
\hline Weight $(\mathrm{kg})^{2}$ & $81.8 \pm 12.1$ & $1.9 \pm 0.2$ \\
\hline BSA $^{\mathrm{a}}\left(\mathrm{m}^{2}\right)$ & $2.0 \pm 0.2$ & \\
\hline Ejection fraction $(\%)$ & & 49 \\
\hline$>50(n)$ & 49 & 2 \\
\hline $30-50(n)$ & 2 & 0 \\
\hline$<30(n)$ & 0 & \\
\hline
\end{tabular}

Abbreviation: BSA, body surface area.

${ }^{\text {a }}$ Results are presented as mean \pm standard deviation. 
Table 2 Baseline

\begin{tabular}{|c|c|c|c|}
\hline & CEPM Ease $(n=51)$ & SJM Trifecta $(n=51)$ & $p$-Value \\
\hline Sinus rhythm & $50(98.0)$ & $48(94.1)$ & \multirow[t]{2}{*}{$0.652^{\mathrm{a}}$} \\
\hline Atrial fibrillation & $1(2.0)$ & $3(5.9)$ & \\
\hline Previous cardiac surgery & $0(0.0)$ & $2(3.9)$ & $0.238^{\mathrm{b}}$ \\
\hline Renal failure & $2(3.9)$ & $1(2.0)$ & $1.000^{\mathrm{a}}$ \\
\hline COPD & $2(3.9)$ & $7(13.7)$ & $0.219^{\mathrm{a}}$ \\
\hline Cerebrovascular attack & $0(0.0)$ & $1(2.0)$ & $0.495^{\mathrm{b}}$ \\
\hline Diabetes mellitus & $10(19.6)$ & $12(23.5)$ & $0.804^{\mathrm{a}}$ \\
\hline Hyperlipidemia & $34(66.7)$ & $39(76.5)$ & $0.302^{\mathrm{a}}$ \\
\hline Systemic hypertension & $34(66.7)$ & $43(84.3)$ & $0.077^{\mathrm{a}}$ \\
\hline Coronary artery disease & $26(51.0)$ & $25(49.0)$ & $0.839^{\mathrm{a}}$ \\
\hline Acute endocarditis & $0(0.0)$ & $4(7.8)$ & \multirow[t]{2}{*}{$0.132^{\mathrm{b}}$} \\
\hline Healed endocarditis & $3(5.9)$ & $1(2.0)$ & \\
\hline NYHA I-II & $34(66.7)$ & $17(33.3)$ & \multirow[t]{2}{*}{$0.000^{\mathrm{b}}$} \\
\hline NYHA III-IV & $14(27.5)$ & $31(60.8)$ & \\
\hline Aortic stenosis & $11(21.6)$ & $20(39.2)$ & \multirow[t]{4}{*}{$0.021^{b}$} \\
\hline Aortic regurgitation & $3(5.9)$ & $0(0.0)$ & \\
\hline Combined aortic lesion & $37(72.5)$ & $29(56.9)$ & \\
\hline Prosthetic valve endocarditis & $0(0.0)$ & $2(3.9)$ & \\
\hline
\end{tabular}

Abbreviations: COPD, chronic obstructive pulmonary disease; NYHA, New York Heart Association.

Note: Results as frequencies (percentage).

a McNemar test.

${ }^{\mathrm{b}}$ Fisher exact test.

Valve-related events occurred in 12 patients (24.5\%) of the CEPM Ease and 7 patients (13.7\%) of the SJM Trifecta group. Structural valve deterioration was not observed in either group. There were two patients with a trivial paravalvular leakage in the SJM Trifecta group.

\section{Thromboembolism}

Thromboembolism occurred in three patients in the CEPM Ease group and one patient in the SJM Trifecta group (McNemar test; $p=0.625$ ). Freedom from thromboembolism at 1 year was $92.5 \%$ (95\% CI [84.5\%; 100\%]) in the CEPM Ease

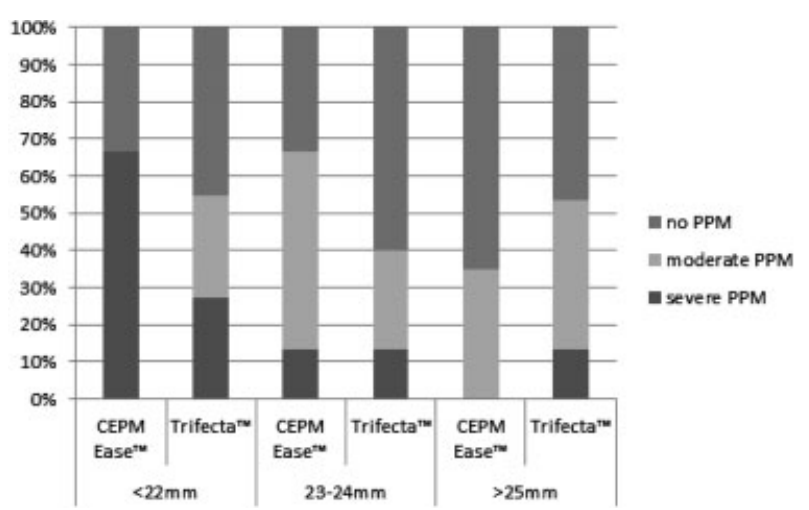

Fig. 1 Kaplan-Meier survival curves at 1 year after the operation. group and 98.0\% (95\% CI [94.0\%; 100\%]) in the SJM Trifecta group, respectively.

\section{Bleeding Events}

Bleeding events were detected in seven patients in the CEPM Ease group and in four patients in the SJM Trifecta group (McNemar-Bowker test; $p=0.607$ ). One case in each group exhibited a major bleeding, requiring hospitalization and blood transfusion. Freedom from major bleeding events at 1 year was $97.9 \%$ (95\% CI [94.0\%; 100\%]) in the CEPM Ease group and $100 \%$ in the SJM Trifecta group, respectively.

\section{Neurological Events}

Three patients suffered from a transient ischemic attack and one patient experienced a stroke in the CEPM Ease group. One patient suffered from a stroke in the SJM Trifecta group. No statistically significant difference in stroke incidence was observed (Fisher's exact test; $p=0.178$ ).

\section{Prosthetic Valve Endocarditis}

The McNemar test revealed no statistically significant difference between the two groups concerning prosthetic valve endocarditis ( $p=0.375$ ). During follow-up, we observed two cases of prosthetic valve endocarditis in the CEPM Ease group. Both patients died within 3 months after the initial operation. One of these patients received a valve replacement and the 
Table 3 Operative data

\begin{tabular}{|c|c|c|c|}
\hline & CEPM Ease $(n=51)$ & Trifecta $(n=51)$ & $p$-Value \\
\hline Median sternotomy ${ }^{a}$ & $20(39.2)$ & $26(51.0)$ & \multirow[t]{2}{*}{$0.286^{\mathrm{b}}$} \\
\hline Partial sternotomy ${ }^{\mathrm{a}}$ & $31(60.8)$ & $25(49.0)$ & \\
\hline Aortic cross-clamp time ${ }^{c}$ & $69.1 \pm 18.9$ & $82.1 \pm 27.3$ & $0.005^{\mathrm{d}}$ \\
\hline Cardiopulmonary bypass time ${ }^{c}$ & $95.4 \pm 26.4$ & $113.5 \pm 35.5$ & $0.001^{\mathrm{d}}$ \\
\hline Concomitant procedures $^{\mathrm{a}}$ & $17(33.3)$ & $24(47.1)$ & $0.167^{\mathrm{e}}$ \\
\hline Coronary artery bypass grafting ${ }^{a}$ & $15(29.4)$ & $18(35.3)$ & $0.648^{\mathrm{e}}$ \\
\hline Mitral valve repair/replacement ${ }^{\mathrm{a}}$ & $0(0.0)$ & $3(5.9)$ & $0.243^{f}$ \\
\hline Tricuspid valve repair/replacement ${ }^{\mathrm{a}}$ & $1(2.0)$ & $1(2.0)$ & $1.000^{f}$ \\
\hline Aortic root enlargement $^{\mathrm{a}}$ & $0(0.0)$ & $1(2.0)$ & $1.000^{f}$ \\
\hline Aortic annulus reconstruction $^{a}$ & $0(0.0)$ & $2(3.9)$ & $0.495^{f}$ \\
\hline Ascending aortic replacement ${ }^{\mathrm{a}}$ & $1(2.0)$ & $0(0.0)$ & $1.000^{f}$ \\
\hline Annulus tissue diameter $(\mathrm{Hegar})^{c}$ & $24.44 \pm 1.82$ & $23.85 \pm 2.17$ & $0.279^{d}$ \\
\hline
\end{tabular}

${ }^{\mathrm{a}}$ Number (percent).

${ }^{\mathrm{b}}$ McNemar-Bowker test.

${ }^{\mathrm{C}}$ Results as mean \pm standard deviation.

dWilcoxon signed-rank test.

${ }^{\mathrm{e}} \mathrm{McNemar}$ test.

${ }^{\mathrm{f}}$ Fisher exact test.

other patient was treated with antibiotics. One case of prosthetic valve endocarditis in the SJM Trifecta group was treated with antibiotics.

\section{Hemodynamic Results}

Hemodynamic results are shown in - Tables $\mathbf{4}$ and $\mathbf{5}$.

\section{Patient-Prosthesis Mismatch}

No statistically significant difference in the incidence of moderate or severe PPM was observed between the two bioprostheses (McNemar-Bowker test, $p=0.873$ ). The incidence of moderate or severe PPM is shown in - Fig. 2.

Table 4 Hemodynamic results grouped by aortic tissue annulus diameter early postoperatively

\begin{tabular}{|c|c|c|c|c|c|c|}
\hline & & CEPM Ease & & SJM Trifecta & & $p$-Value \\
\hline \multirow[t]{3}{*}{$<22 \mathrm{~mm}$} & MPG early postop & $\begin{array}{l}(n=4) \\
18.02 \pm 4.96\end{array}$ & $17.80(13.28-23.20)$ & $\begin{array}{l}(n=12) \\
7.21 \pm 4.58\end{array}$ & $6.45(1.00-14.57)$ & $0.016^{\mathrm{a}}$ \\
\hline & EOA early postop & $1.30 \pm 0.46$ & $1.17(0.91-1.97)$ & $1.74 \pm 0.50$ & $1.67(1.18-2.81)$ & $0.214^{\mathrm{a}}$ \\
\hline & EOAI early postop & $0.75 \pm 0.21$ & $0.69(0.56-1.05)$ & $1.00 \pm 0.34$ & $0.91(0.65-1.72)$ & $0.214^{\mathrm{a}}$ \\
\hline \multirow[t]{3}{*}{$23-24 \mathrm{~mm}$} & MPG early postop & $\begin{array}{l}(n=18) \\
11.50 \pm 3.01\end{array}$ & $11.73(7.50-19.00)$ & $\begin{array}{l}(n=19) \\
6.27 \pm 4.05\end{array}$ & $5.60(0.40-12.50)$ & $0.001^{a}$ \\
\hline & EOA early postop & $1.87 \pm 0.43$ & 1.89 (1.30-2.94) & $2.21 \pm 0.5$ & $2.14(1.37-3.38)$ & $0.056^{\mathrm{a}}$ \\
\hline & EOAI early postop & $0.95 \pm 0.22$ & $0.98(0.63-1.28)$ & $1.13 \pm 0.29$ & $1.06(0.63-1.72)$ & $0.148^{\mathrm{a}}$ \\
\hline \multirow[t]{3}{*}{$>25 \mathrm{~mm}$} & MPG early postop & $\begin{array}{l}(n=24) \\
12.09 \pm 3.62\end{array}$ & $11.30(6.90-18.80)$ & $\begin{array}{l}(n=16) \\
7.14 \pm 2.43\end{array}$ & $7.00(3.60-11.50)$ & $0.000^{\mathrm{a}}$ \\
\hline & EOA early postop & $1.85 \pm 0.46$ & $1.86(0.97-2.76)$ & $1.99 \pm 0.51$ & $2.02(0.96-2.70)$ & $0.370^{\mathrm{a}}$ \\
\hline & EOAI early postop & $0.92 \pm 0.21$ & $0.94(0.53-1.35)$ & $0.97 \pm 0.22$ & $1.03(0.48-1.28)$ & $0.327^{a}$ \\
\hline \multirow[t]{3}{*}{ All sizes } & MPG early postop & $\begin{array}{l}(n=50) \\
12.11 \pm 3.80\end{array}$ & $11.77(6.67-23.20)$ & $\begin{array}{l}(n=51) \\
6.95 \pm 4.06\end{array}$ & $6.70(0.40-18.30)$ & $0.000^{\mathrm{b}}$ \\
\hline & EOA early postop & $1.80 \pm 0.44$ & $1.87(0.91-2.94)$ & $2.03 \pm 0.50$ & $1.99(0.96-3.38)$ & $0.047^{b}$ \\
\hline & EOAI early postop & $0.92 \pm 0.21$ & $0.94(0.53-1-35)$ & $1.04 \pm 0.28$ & $1.04(0.48-1.72)$ & $0.032^{\mathrm{b}}$ \\
\hline
\end{tabular}

Abbreviations: EOA, effective orifice area $\left(\mathrm{cm}^{2}\right)$; EOAI, effective orifice area index $\left(\mathrm{cm}^{2} / \mathrm{m}^{2}\right)$; MPG, mean pressure gradient $(\mathrm{mm} \mathrm{Hg})$. Note: Results are presented as mean \pm standard deviation and median (range).

${ }^{a}$ Mann-Whitney $U$ test.

${ }^{b}$ Wilcoxon signed-rank test. 
Table 5 Hemodynamic results grouped by aortic tissue annulus diameter 1 year postoperatively

\begin{tabular}{|c|c|c|c|c|c|c|}
\hline \multirow{4}{*}{$<22 \mathrm{~mm}$} & \multirow[b]{2}{*}{ MPG 1-y postop } & \multicolumn{2}{|l|}{ CEPM Ease } & \multicolumn{2}{|l|}{ SJM Trifecta } & \multirow{2}{*}{$\frac{p \text {-Value }}{0.038^{\mathrm{a}}}$} \\
\hline & & $\begin{array}{l}(n=4) \\
17.73 \pm 4.51\end{array}$ & $16.73(13.80-22.66)$ & $\begin{array}{l}(n=12) \\
10.01 \pm 4.3\end{array}$ & $9.00(3.00-16.00)$ & \\
\hline & EOA 1-y postop & $1.16 \pm 0.30$ & $1.06(0.92-1.50)$ & $1.49 \pm 0.29$ & $1.48(0.98-1.89)$ & $0.170^{\mathrm{a}}$ \\
\hline & EOAI 1-y postop & $0.69 \pm 0.22$ & $0.57(0.55-0.95)$ & $0.83 \pm 0.21$ & $0.84(0.48-1.16)$ & $0.368^{\mathrm{a}}$ \\
\hline \multirow[t]{3}{*}{$23-24 \mathrm{~mm}$} & MPG 1-y postop & $\begin{array}{l}(n=16) \\
13.27 \pm 4.25\end{array}$ & $12.53(8.70-25.95)$ & $\begin{array}{l}(n=18) \\
10.64 \pm 5.18\end{array}$ & $10.00(4.00-20.00)$ & $0.202^{\mathrm{a}}$ \\
\hline & EOA 1-y postop & $1.64 \pm 0.48$ & $1.47(1.06-2.71)$ & $1.75 \pm 0.42$ & $1.68(1.10-2.68)$ & $0.250^{\mathrm{a}}$ \\
\hline & EOAI 1-y postop & $0.82 \pm 0.20$ & $0.79(0.51-1.25)$ & $0.91 \pm 0.25$ & $0.87(0.53-1.58)$ & $0.202^{\mathrm{a}}$ \\
\hline \multirow[t]{3}{*}{$>25 \mathrm{~mm}$} & MPG 1-y postop & $\begin{array}{l}(n=23) \\
11.77 \pm 3.17\end{array}$ & $11.8(6.49-18.24)$ & $\begin{array}{l}(n=16) \\
8.01 \pm 2.82\end{array}$ & $8.00(3.00-13.00)$ & $0.002^{\mathrm{a}}$ \\
\hline & EOA 1-y postop & $1.83 \pm 0.34$ & $1.74(1.37-2.61)$ & $1.70 \pm 0.40$ & $1.69(1.20-2.51)$ & $0.314^{\mathrm{a}}$ \\
\hline & EOAI 1-y postop & $0.92 \pm 0.16$ & $0.91(0.68-1.27)$ & $0.84 \pm 0.21$ & $0.84(0.60-1.26)$ & $0.107^{a}$ \\
\hline \multirow[t]{3}{*}{ All sizes } & MPG 1-y postop & $\begin{array}{l}(n=47) \\
12.86 \pm 3.80\end{array}$ & $12.90(6.49-25.95)$ & $\begin{array}{l}(n=49) \\
9.40 \pm 4.27\end{array}$ & $8.00(3.00-20.00)$ & $0.001^{b}$ \\
\hline & EOA 1-y postop & $1.69 \pm 0.42$ & $1.64(0.92-2.71)$ & $1.66 \pm 0.39$ & $1.64(0.98-2.68)$ & $0.839^{b}$ \\
\hline & EOAI 1-y postop & $0.86 \pm 0.18$ & $0.86(0.51-1.27)$ & $0.86 \pm 0.22$ & $0.85(0.48-1.58)$ & $0.689^{b}$ \\
\hline
\end{tabular}

Abbreviations: EOA, effective orifice area $\left(\mathrm{cm}^{2}\right)$; EOAl, effective orifice area index $\left(\mathrm{cm}^{2} / \mathrm{m}^{2}\right)$; MPG, mean pressure gradient $(\mathrm{mm} \mathrm{Hg})$. Note: Results are presented as mean \pm standard deviation and median (range).

aMann-Whitney $U$ test.

bWilcoxon signed-rank test.

\section{Left Ventricular Mass Regression}

A significant LVM regression was detected in both groups 1 year after surgery (Wilcoxon signed-rank test; $p=0.000$ for the CEPM Ease and $p=0.047$ for the Trifecta valve), without any statistically significant difference between the two prostheses (Wilcoxon signed-rank test; $p=0.929$ pre-op and $p=0.723$ at 1 year).

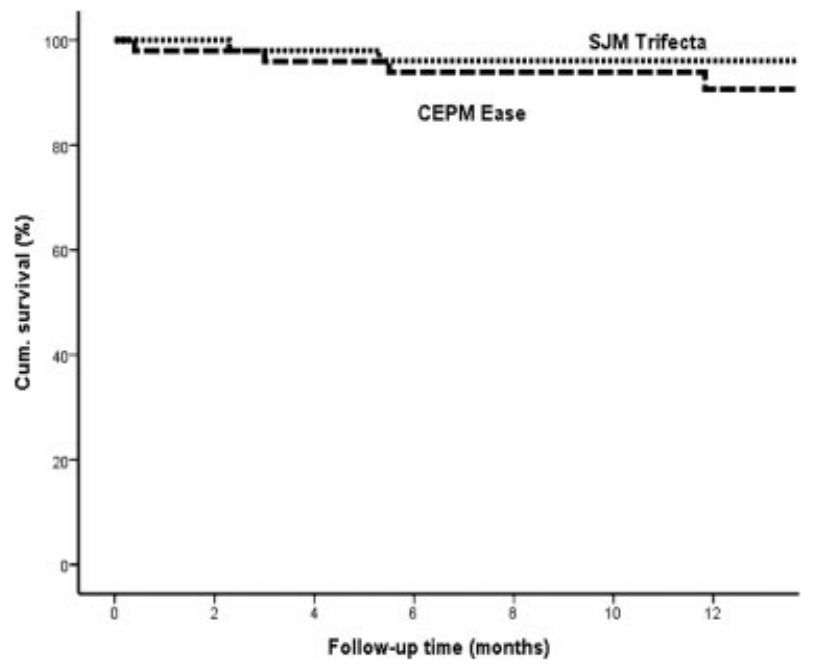

Fig. 2 PPM grouped by aortic tissue annulus diameter 1 year after the operation. Trifecta, $n=41$; CEPM Ease, $n=38$. PPM, patient-prosthesis mismatch.

\section{Discussion}

The main findings of the study are as follows.

- The SJM Trifecta bioprosthesis shows lower MPGs in small ( $\leq 22 \mathrm{~mm}$ ), medium (23-24 mm), and large ( $\geq 25 \mathrm{~mm})$ annulus sizes early postoperatively and lower MPGs in small $(\leq 22 \mathrm{~mm})$ and large $(\geq 25 \mathrm{~mm})$ annulus sizes at 1 year, when compared with the CEPM Ease bioprosthesis.

- The SJM Trifecta bioprosthesis shows higher EOA and EOAI across all annulus sizes (18-29 mm) early postoperatively. With regard to EOA and EOAI, no statistically significant differences between the two groups across all annulus sizes $(18-29 \mathrm{~mm})$ at 1 year and no differences in the different annulus sizes ( $\leq 22,23-24$, and $\geq 25 \mathrm{~mm}$ ) early postoperatively and at 1 year were observed.

- No statistically significant differences between the two groups with regard to LVM regression and PPM were observed.

- Survival and valve-related complications, such as prosthetic valve endocarditis, major bleeding, and thromboembolic events, are comparable between the two groups.

To date, only two studies have compared hemodynamic data of the new SJM Trifecta valve with bioprostheses of other manufacturers. In a nonrandomized, observational study of Wendt et al, 346 consecutive patients after AVR with the SJM Trifecta, the Carpentier-Edwards Perimount Magna, or the CEPM Ease, bioprostheses were analyzed. ${ }^{17}$ In a multivariate covariance analysis after 6 months, Wendt et al could demonstrate no influence of prosthesis type on MPG and aortic 
valve area. ${ }^{17}$ In contrast, Ugur et al showed in a retrospective study of 1,436 patients after AVR with the SJM Trifecta, the Sorin Mitroflow (Milan, Italy), or the Carpentier-Edwards Perimount Magna bioprostheses early postoperative a favorable hemodynamic performance of the SJM Trifecta valve. ${ }^{18}$ In both studies, the prostheses were compared on the basis of their size, labeled by the manufacturer. ${ }^{17,18}$ However, this method of comparing different valve prostheses may be controversial. In previous studies, we described that geometric dimensions of prostheses of the same labeled size of different manufactures vary widely and are thus no basis for the comparison of their hemodynamic performance. ${ }^{19-21}$

To compare the hemodynamic performance of different bioprostheses, we propose to refer the results to the "true" aortic tissue annulus diameter. We therefore used a Hegar dilator to measure the aortic tissue annulus diameter intraoperatively after decalcification of the annulus and prior to the implantation of the prosthesis.

\section{Hemodynamic Results}

Both bioprostheses analyzed in our study showed excellent hemodynamics early postoperatively and at 1 year, and are comparable to the results of Wendt et al and Bavaria et al. ${ }^{17,22}$ The superiority in hemodynamics of the SJM Trifecta might be due to the new concept of valve design chosen by St. Jude Medical. The titanium stent of the SJM Trifecta valve is thinner than the cobalt-chromium stent of the CEPM Ease valve. In addition, the stent covering of the SJM Trifecta, which is made of a thin polyester layer and porcine pericardium, contributes to the slenderness of the stent posts. In contrast to the CEPM Ease, the leaflets of the Trifecta are mounted exterior of the sewing ring, so that they can open more fully and the thickness of the leaflets is less important. All these factors are contributing to a relatively larger internal diameter of the SJM Trifecta prostheses.

However, no statistically significant differences with regard to the EOAs and EOAIs between the two groups across all annulus sizes at 1 year and no statistically significant differences in the different annulus sizes $(\leq 22,23-24$, and $\geq 25$ $\mathrm{mm}$ ), neither postoperatively nor at 1 year, could be observed.

\section{Survival}

The 30-day mortality rate of the present investigation is comparable with the SJM Trifecta multicenter, prospective clinical trial of Bavaria et al with an early ( $\leq 30$ days) mortality of $1.8 \%$ in 1,014 patients. ${ }^{22}$ Wyss et al report about an inhospital mortality of $2.2 \%$ after AVR with either the CEPM Ease bioprosthesis or the Carpentier-Edwards Perimount Magna bioprosthesis in 270 patients. ${ }^{9}$ In the retrospective study of Wendt et al on 346 consecutive patients, 30-day mortalities in the SJM Trifecta, the Carpentier-Edwards Perimount Magna, and the CEPM Ease group were 8.3, 15, and $7.1 \%$, respectively. The higher mortality in this cohort might be due to their older patient collective (66 vs. $72-75$ years) and a higher rate of concomitant procedures, such as aortic root enlargement. ${ }^{17}$ Aortic root enlargement was performed in the study of Wendt et al in the SJM Trifecta group in 9.1\%, in the Carpentier-Edwards Perimount Magna group in 25.4\%, and in the CEPM Ease group in $12.1 \%$, respectively. ${ }^{17}$

Concerning the SJM Trifecta group, the survival rate in the present investigation is comparable with the data of the SJM Trifecta multicenter, prospective clinical trial of Bavaria et al with an overall survival rate of $95.8 \%$ at 1 year. ${ }^{22}$ In our CEPM Ease cohort, the overall survival at 1 year compares favorably to the data of Dalmau et al, who describe an overall survival of $100 \%$ at 1 year in 54 patients. ${ }^{16}$

\section{Patient-Prosthesis Mismatch}

As shown previously by Blais et al, PPM represents a strong and independent risk factor for short-term mortality and its prevalence after AVR varies between 19 and $70 \% .{ }^{23}$ In our SJM Trifecta cohort, the incidence of moderate and severe PPM was 31.7 and $17.1 \%$ across all annulus sizes, respectively. In the CEPM Ease group, the incidence of moderate and severe PPM was 39.5 and $10.5 \%$, respectively. Although MPGs were lower and EOAs were higher in the SJM Trifecta group, no statistically significant difference between the two groups with regard to moderate or severe PPM could be observed.

In the study of Wendt et al, moderate PPM was $21 \%$ in the SJM Trifecta group, 23\% in the Carpentier-Edwards Perimount Magna $^{\mathrm{TM}}$ group, and $1 \%$ in the CEPM Ease group $(p<0.01)$. Severe PPM was not present in all 346 patients. ${ }^{17}$ In comparison to our results, the low incidence of moderate PPM and the absence of severe PPM might be due to the fact that aortic root enlargement was performed in 9 to $25 \%$ of their patients. Concerning their higher 30-day mortality (up to 15\%), one might speculate that aortic root enlargement and the consecutive avoidance of PPM are also associated with a higher 30-day mortality rate. ${ }^{17}$ As shown previously by Ruzicka et al, even with aortic valve prostheses designed for complete supra-annular implantation, PPM is not completely avoidable in all patients. ${ }^{6}$ The choice of a bovine prosthesis can optimize hemodynamic performance, as moderate and severe PPM is more frequent when using a porcine prosthesis. ${ }^{6}$ Within decision making in case of PPM, the surgeon has to balance between a higher operative mortality in case of aortic root enlargement and a higher short-term mortality in case of moderate or severe PPM. ${ }^{17,23}$

\section{Left Ventricular Mass Regression}

LV hypertrophy is an independent risk factor for morbidity and mortality. ${ }^{24}$

To date, there are no publications available dealing with the issue of LVM regression after AVR with either the CEPM Ease or the SJM Trifecta biological prostheses. Our results are comparable to the retrospective study of Tasca et al, who examined 88 consecutive patients undergoing AVR with the Carpentier-Edwards Perimount bioprosthesis in small aortic annuli. ${ }^{14}$ The authors report of a mean LVM regression ranging from 19.3 to $21.9 \%$ ( $p=0.860) 1$ year after the operation. ${ }^{14}$

Despite a significant LVM regression in our cohort, LV hypertrophy was still present in 33.3\% of the CEPM Ease patients and in $52.2 \%$ of the Trifecta patients. In accordance with our data, the study of Tasca et al found that residual left ventricular hypertrophy was present in 44 to $68 \%{ }^{14}$ 


\section{Limitations}

In the present nonrandomized retrospective study, the follow-up is short. Due to the short-term follow-up, the impact of the new design concept of the SJM Trifecta bioprosthesis on mid- or long-term performance was not studied. There is a possible bias because of the low number of patients in the subgroups according to the native aortic annulus. Maybe a propensity score-based matching including all patients (201 vs. 61) would have been more appropriate. Stress echocardiography was not performed.

\section{Conclusion}

The SJM Trifecta valve showed lower MPGs early postoperative and at 1 year as well as higher EOA and EOAI early postoperative when compared with the CEPM Ease bioprosthesis. No significant differences were detected with regard to LVM regression and PPM. Severe PPM and valve-related adverse events were infrequent overall.

\section{Conflict of Interest}

None declared.

\section{Acknowledgments}

This work was not supported by grants, financial support, and technical or other assistance.

\section{References}

1 Vahanian A, Alfieri O, Andreotti F, et al; Joint Task Force on the Management of Valvular Heart Disease of the European Society of Cardiology (ESC); European Association for Cardio-Thoracic Surgery (EACTS). Guidelines on the management of valvular heart disease (version 2012). Eur Heart J 2012;33(19):2451-2496

2 Funkat AK, Beckmann A, Lewandowski J, et al. Cardiac surgery in Germany during 2011: a report on behalf of the German Society for Thoracic and Cardiovascular Surgery. Thorac Cardiovasc Surg 2012;60(6):371-382

3 El Oakley R, Kleine P, Bach DS. Choice of prosthetic heart valve in today's practice. Circulation 2008;117(2):253-256

4 Totaro P, Degno N, Zaidi A, Youhana A, Argano V. CarpentierEdwards PERIMOUNT Magna bioprosthesis: a stented valve with stentless performance? J Thorac Cardiovasc Surg 2005;130(6): $1668-1674$

5 Guenzinger R, Eichinger WB, Botzenhardt F, et al. Rest and exercise performance of the Medtronic Advantage bileaflet valve in the aortic position. Ann Thorac Surg 2005;80(4):1319-1326

6 Ruzicka DJ, Hettich I, Hutter A, et al. The complete supraannular concept: in vivo hemodynamics of bovine and porcine aortic bioprostheses. Circulation 2009;120(11, Suppl):S139-S145

7 Wagner IM, Eichinger WB, Bleiziffer S, et al. Influence of completely supra-annular placement of bioprostheses on exercise hemodynamics in patients with a small aortic annulus. J Thorac Cardiovasc Surg 2007;133(5):1234-1241

8 Dalmau MJ, Maríagonzález-Santos J, López-Rodríguez J, Bueno M, Arribas A. The Carpentier-Edwards Perimount Magna aortic xenograft: a new design with an improved hemodynamic performance. Interact Cardiovasc Thorac Surg 2006;5(3):263-267

9 Wyss TR, Bigler M, Stalder M, et al. Absence of prosthesis-patient mismatch with the new generation of Edwards stented aortic bioprosthesis. Interact Cardiovasc Thorac Surg 2010;10(6): 884-887, discussion 887-888

10 Edmunds LH Jr, Clark RE, Cohn LH, Grunkemeier GL, Miller DC, Weisel RD; Ad Hoc Liaison Committee for Standardizing Definitions of Prosthetic Heart Valve Morbidity of The American Association for Thoracic Surgery and The Society of Thoracic Surgeons. Guidelines for reporting morbidity and mortality after cardiac valvular operations. J Thorac Cardiovasc Surg 1996;112(3): 708-711

11 Nolan SP. The International Standard Cardiovascular implantsCardiac Valve Prostheses (ISO 5840:1989) and the FDA Draft Replacement Heart Valve Guidance (Version 4.0). J Heart Valve Dis 1994;3(4):347-349

12 Du Bois D, Du Bois EF. A formula to estimate the approximate surface area if height and weight be known. 1916. Nutrition 1989; 5(5):303-311, discussion 312-313

13 Devereux RB, Alonso DR, Lutas EM, et al. Echocardiographic assessment of left ventricular hypertrophy: comparison to necropsy findings. Am J Cardiol 1986;57(6):450-458

14 Tasca G, Brunelli F, Cirillo $M$, et al. Mass regression in aortic stenosis after valve replacement with small size pericardial bioprosthesis. Ann Thorac Surg 2003;76(4):1107-1113

15 Pibarot P, Dumesnil JG. Hemodynamic and clinical impact of prosthesis-patient mismatch in the aortic valve position and its prevention. J Am Coll Cardiol 2000;36(4):1131-1141

16 Dalmau MJ, González-Santos JM, Blázquez JA, et al. Hemodynamic performance of the Medtronic Mosaic and Perimount Magna aortic bioprostheses: five-year results of a prospectively randomized study. Eur J Cardiothorac Surg 2011;39(6):844-852, discussion 852

17 Wendt D, Thielmann M, Plicht B, et al. The new St Jude Trifecta versus Carpentier-Edwards Perimount Magna and Magna Ease aortic bioprosthesis: is there a hemodynamic superiority? J Thorac Cardiovasc Surg 2014;147(5):1553-1560

18 Ugur M, Suri RM, Daly RC, et al. Comparison of early hemodynamic performance of 3 aortic valve bioprostheses. J Thorac Cardiovasc Surg 2014;148(5):1940-1946

19 Botzenhardt F, Eichinger WB, Bleiziffer S, et al. Hemodynamic comparison of bioprostheses for complete supra-annular position in patients with small aortic annulus. J Am Coll Cardiol 2005; 45(12):2054-2060

20 Eichinger WB, Botzenhardt F, Wagner I, et al. Hemodynamic evaluation of the Sorin Soprano bioprosthesis in the completely supra-annular aortic position. J Heart Valve Dis 2005;14(6): 822-827

21 Guenzinger R, Eichinger WB, Hettich I, et al. A prospective randomized comparison of the Medtronic Advantage Supra and St Jude Medical Regent mechanical heart valves in the aortic position: is there an additional benefit of supra-annular valve positioning? J Thorac Cardiovasc Surg 2008;136(2):462-471

22 Bavaria JE, Desai ND, Cheung A, et al. The St Jude Medical Trifecta aortic pericardial valve: results from a global, multicenter, prospective clinical study. J Thorac Cardiovasc Surg 2014;147(2): 590-597

23 Blais C, Dumesnil JG, Baillot R, Simard S, Doyle D, Pibarot P. Impact of valve prosthesis-patient mismatch on short-term mortality after aortic valve replacement. Circulation 2003;108(8):983-988

24 Levy D, Garrison RJ, Savage DD, Kannel WB, Castelli WP. Prognostic implications of echocardiographically determined left ventricular mass in the Framingham Heart Study. N Engl J Med 1990;322(22): 1561-1566 